\title{
Infecção por SARS-CoV-2 em pacientes neonatos: aspectos gerais
}

\author{
SARS-CoV-2 Infection in Neonatal Patients: general aspects \\ Infección por SARS-CoV-2 en pacientes neonatales: aspectos generales
}

Recebido: 01/09/2021 | Revisado: 08/09/2021 | Aceito: 10/09/2021 | Publicado: 13/09/2021

Lennara Pereira Mota

ORCID: https://orcid.org/0000-0002-2629-6634

Centro Universitário Maurício de Nassau, Brasil

E-mail: lennaramota@hotmail.com

Josefa Angélica Cerqueira Poty

ORCID: https://orcid.org/0000-0002-3838-0833

Centro Universitário UniFacid, Brasil

E-mail: potyjosefa@gmail.com

Talissa Brenda de Castro Lopes

ORCID: https://orcid.org/0000-0001-7694-1067

Universidade Federal do Piauí, Brasil

E-mail: talissa.brenda@gmail.com

Marcello de Alencar Silva

ORCID: https://orcid.org/0000-0001-9451-2979

Centro Universitário UniFacid, Brasil

E-mail: marcello.silva@professores.facid.edu.br

Emanuelle Paiva de Vasconcelos Dantas

ORCID: https://orcid.org/0000-0001-7752-0416

Centro Universitário Uninassau, Brasil

E-mail: emanuellepaiva@yahoo.com.br

Vitor Kauê de Melo Alves

ORCID: https://orcid.org/0000-0002-7283-0850 Universidade Estadual do Piauí, Brasil

E-mail: vitorkaue_@hotmail.com

Mickaelle Bezerra Calaça

ORCID: https://orcid.org/0000-0001-9872-6675

Universidade Estadual do Maranhão, Brasil

E-mail: mickaellebezerra@outlook.com

Alaíde Silva Lemos

ORCID: https://orcid.org/0000-0003-4121-6724

Centro Universitário Uninovafapi, Brasil

E-mail: laid_mel13@hotmail.com

Sangella da Silva Soares

ORCID: https://orcid.org/0000-0003-1592-7207

Centro Universitário Uninovafapi, Brasil

E-mail: sangella_soares@hotmail.com

Vânia da Silva Vidal

ORCID: https://orcid.org/0000-0001-5571-3911 Universidade Federal do Piauí, Brasil

E-mail: vidals.vania@gmail.com

Thaynara Alves Rodrigues Paulo da Silva

ORCID: https://orcid.org/0000-0002-8268-9627

Centro Universitário Uninovafapi, Brasil

E-mail: Thaynara.Alves.biom@gmail.com

Isadora Alencar da Silva Andrade

ORCID: https://orcid.org/0000-0002-0195-4514

Centro Universitário Uninovafapi, Brasil

E-mail: Isadoraandrade733@gmail.com

Luciana Alencar Fialho Bringel

ORCID: https://orcid.org/0000-0001-6004-5727

Universidade Federal do Maranhão, Brasil

E-mail: luciana_bringel@hotmail.com

Yaskara Raissa de Pádua Sampaio

ORCID: https://orcid.org/0000-0001-5983-2387

Centro Universitário Uninovafapi, Brasil

E-mail: Yaskararaissa@gmail.com

Sérgio Henrique Assunção Lacerda Borges

ORCID: https://orcid.org/0000-0002-8553-7223

Centro Universitário Uninovafapi, Brasil

E-mail: seergiol@hotmail.com 


\author{
João Victor da Cunha Silva \\ ORCID: https://orcid.org/0000-0002-1934-7595 \\ Universidade Federal do Maranhão, Brasil \\ E-mail: joaovictor.csilva@ hotmail.com \\ Cristiane de Souza Pantoja \\ ORCID: https://orcid.org/0000-0002-9681-402X \\ Universidade da Amazônia, Brasil \\ E-mail: contatocristianesouza@outlook.com \\ Adriano Correia de Sousa \\ ORCID: https://orcid.org/0000-0002-9510-0965 \\ Centro Universitário de Ciências e Tecnologia do Maranhão, Brasil \\ E-mail: Adrianodesousa@outlook.com.br \\ Matheus Pedrosa de Oliveira \\ ORCID: https://orcid.org/0000-0003-4003-3184 \\ Universidade Federal do Delta do Parnaíba, Brasil \\ E-mail: matheus_pedrosasete@hotmail.com
}

\begin{abstract}
Resumo
Até o momento as evidencias sugerem que o risco de o recém-nascido (RN) ser infectado durante a hospitalização do parto é baixo, porém apenas quando tem-se os cuidados necessários para proteger esses pacientes de secreções respiratórias infecciosas da mãe e quando são realizadas medidas de prevenção no quarto e na amamentação. O trabalho tem por objetivo expor através da análise de artigos científicos os principais aspectos envolvidos na infecção por SARS-CoV-2 em pacientes neonatos. Trata-se de uma revisão bibliográfica de caráter qualitativo e sistemático realizada entre os anos de 2019 a 2021. Há uma preocupação levantada sobre o risco que a infecção por COVID-19 pode representar para o feto. Se a propagação vertical do vírus é possível e quais consequências ela teria, ainda não está totalmente esclarecido, mas o risco parece relativamente pequeno. Apesar de não ser possível presumir a repercussão para os pacientes neonatais, é possível evitar a mortalidade com adoção de protocolos já estabelecidos. É primordial a busca constante em esclarecer os mecanismos, e as formas de transmissão nesses pacientes, em vista disso, a realização de novos estudos científicos mais completos que abordem esta temática faz-se necessário.
\end{abstract}

Palavras-chave: Covid-19; Recém-nascido; Infecção; Prematuridade.

\begin{abstract}
So far, evidence suggests that the risk of the newborn (NB) being infected during hospitalization for childbirth is low, but only when the necessary care is taken to protect these patients from the mother's infectious respiratory secretions and when they are performed prevention measures in the bedroom and breastfeeding. The work aims to expose through the analysis of scientific articles the main aspects involved in SARS-CoV-2 infection in newborn patients. This is a literature review of a qualitative and systematic nature carried out between the years 2019 to 2021 . There is a concern raised about the risk that COVID-19 infection can represent for the fetus. Whether vertical propagation of the virus is possible and what consequences it would have is not entirely clear, but the risk appears relatively small. Although it is not possible to assume the repercussion for neonatal patients, it is possible to avoid mortality by adopting established protocols. It is essential to constantly seek to clarify the mechanisms and forms of transmission in these patients, in view of this, the realization of new, more complete scientific studies that address this issue is necessary.
\end{abstract}

Keywords: Covid-19; Newborn; Infection; Prematurity.

\begin{abstract}
Resumen
Hasta el momento, la evidencia sugiere que el riesgo de infección del recién nacido (RN) durante la hospitalización por parto es bajo, pero solo cuando se toman los cuidados necesarios para proteger a estas pacientes de las secreciones respiratorias infecciosas de la madre y cuando se realizan medidas de prevención en el dormitorio y lactancia. El trabajo tiene como objetivo exponer a través del análisis de artículos científicos los principales aspectos involucrados en la infección por SARS-CoV-2 en pacientes recién nacidos. Se trata de una revisión de la literatura de carácter cualitativo y sistemático realizada entre los años 2019 a 2021. Existe preocupación por el riesgo que puede representar la infección por COVID-19 para el feto. No está del todo claro si es posible la propagación vertical del virus y qué consecuencias tendría, pero el riesgo parece relativamente pequeño. Aunque no es posible asumir la repercusión para los pacientes neonatales, es posible evitar la mortalidad adoptando los protocolos establecidos. Es fundamental buscar constantemente el esclarecimiento de los mecanismos y formas de transmisión en estos pacientes, para ello es necesaria la realización de nuevos estudios científicos más completos que aborden este tema.
\end{abstract}

Palabras clave: COVID-19; Recién nacido; Infección; Precocidad.

\title{
1. Introdução
}

No final de 2019, surgiu na China o novo coronavírus, um vírus que causa doença respiratória aguda grave (SARS- 
CoV-2). Logo, esse vírus e doenças relacionadas, denominado COVID-19 (Doença do Coronavírus 2019), tornou-se uma pandemia global. A rápida disseminação levantou preocupações sobre grupos potencialmente vulneráveis, como por exemplo, mulheres grávidas e recém-nascidos (Rathberger et al., 2021; Cavalcante et al., 2020).

Segundo dados do Ministério da Saúde, cerca de 172 milhões de casos de infecção por coronavírus afetaram a população do mundo. No Brasil, a taxa de mortes estimada é de 1.860 óbitos por milhão de habitantes, em que cerca de 330, ou seja, (12,6\%) são grávidas ou puérperas que foram à óbito por COVID 19. Crianças entre 0 a 5 anos de idade, foram à óbito cerca de 627 indivíduos, sendo que 420 eram bebês entre 0 e 12 meses (Barbosa et al., 2021; Brasil, 2021).

Atualmente as evidencias sugerem que o risco de o recém-nascido (RN) ser infectado durante a hospitalização do parto é baixo, porém apenas quando tem-se os cuidados necessários para proteger esses pacientes de secreções respiratórias infecciosas da mãe e quando são realizadas medidas de prevenção no quarto e na amamentação (Shah; Saugstad, 2021).

Em gestantes infectadas por SARS-CoV-2, é necessária uma atenção redobrada, visto que, estudos mostram que outras doenças endêmicas causadas pelo coronavírus - síndrome respiratória aguda grave (SARS) e síndrome respiratória do oriente médio (MERS) - podem causar, em fetos de mães positivas, crescimento intrauterino restrito (CIUR). Ainda não há estudos que comprovem essa mesma particularidade advinda do agente etiológico da COVID-19. Toda via, tem aumentado o número de partos prematuros em gestantes infectadas, o que esclarece a recomendação de administrar corticoides entre 24 e 34 semanas de gestação em casos de mães com quadro clínico de potencial gravidade (Oliveira, et al., 2021).

O tratamento clínico de forma geral de pacientes recém-nascidos positivos para o SARS-CoV-2 é sintomático e de suporte, em que envolve a manutenção da homeostase, acompanhamento de exames laboratoriais, radiografia de tórax, prevenção e tratamento de possíveis complicações e utilização do suporte respiratório, caso seja necessário. Uma das prioridades é a detecção de problemas respiratórios agudos neonatais de forma a classificar a gravidade clínica e fornecer suporte ventilatório seguindo as diretrizes e melhores evidências disponíveis (Goés et al., 2020).

Durante esta pandemia, o rompimento tardio do cordão umbilical, o contato com a pele e o início da amamentação também chamaram a atenção das pessoas porque afetam a saúde e o desenvolvimento da primeira infância e o atendimento integral. A Organização Mundial de Saúde (OMS) recomenda alimentar os bebês nascidos de mães com infecção suspeita, provável ou confirmada de COVID-19, de acordo com as diretrizes de alimentação infantil padrão, tomando os cuidados necessários para controlar e prevenir a infecção A OMS afirma que sempre que uma mãe ficar gravemente doente devido à COVID-19, ou outras complicações que impossibilitem o cuidado e / ou amamentação de seu bebê, ela deve ser incentivada a retirar o leite materno com segurança e fornecê-lo ao bebê (Duran et al., 2020).

O trabalho tem por objetivo expor através da análise de artigos científicos os principais aspectos envolvidos na infecção por SARS-CoV-2 em pacientes neonatos.

\section{Métodos}

Trata-se de uma revisão bibliográfica de caráter qualitativo e sistemático. A busca pelos textos foi realizada a partir das seguintes palavras-chaves indexadas no DECS (Descritores em Ciências da Saúde): “Covid-19”; "Recém-nascido"; "Infecção" e "Prematuridade" nos idiomas português, inglês e espanhol na plataforma Scientific Electronic Library Online (SCIELO) e Biblioteca Virtual em Saúde (BVS).

Os critérios de inclusão foram pesquisas científicas publicadas de 2019 a 2021, publicados no idioma português, inglês e espanhol, que atendiam ao problema da pesquisa: Quais os principais aspectos envolvidos na infecção por SARS-Cov2 em pacientes neonatos? Os critérios de exclusão foram trabalhos científicos com apenas resumos disponíveis, publicações duplicadas, artigos de relato de experiência, reflexivo, editoriais, comentários e cartas ao editor. 
A partir do problema de pesquisa foram selecionados artigos publicados em periódicos nacionais e internacionais escolhidos a partir de levantamento realizado por meios dos descritores na biblioteca virtual Scientific Electronic Library Online, Biblioteca Virtual em Saúde (BVS) e dissertações e teses.

Os artigos foram selecionados para leitura e foram incluídos na análise e resultados artigos que se enquadravam no tema proposto do trabalho.

\section{Resultados e Discussão}

A Figura 1 ilustra os resultados deste trabalho que foram obtidos através da análise quantitativa de publicações sobre os principais aspectos envolvidos na infecção por SARS-CoV-2 em pacientes neonatos nas bases de dados Scientific Electronic Library Online e Biblioteca Virtual em Saúde (BVS).

Figura 1. Fluxograma que apresenta o processo de seleção das publicações sobre a infecção por SARS-CoV-2 em pacientes neonatos, Teresina, Brasil, 2021.

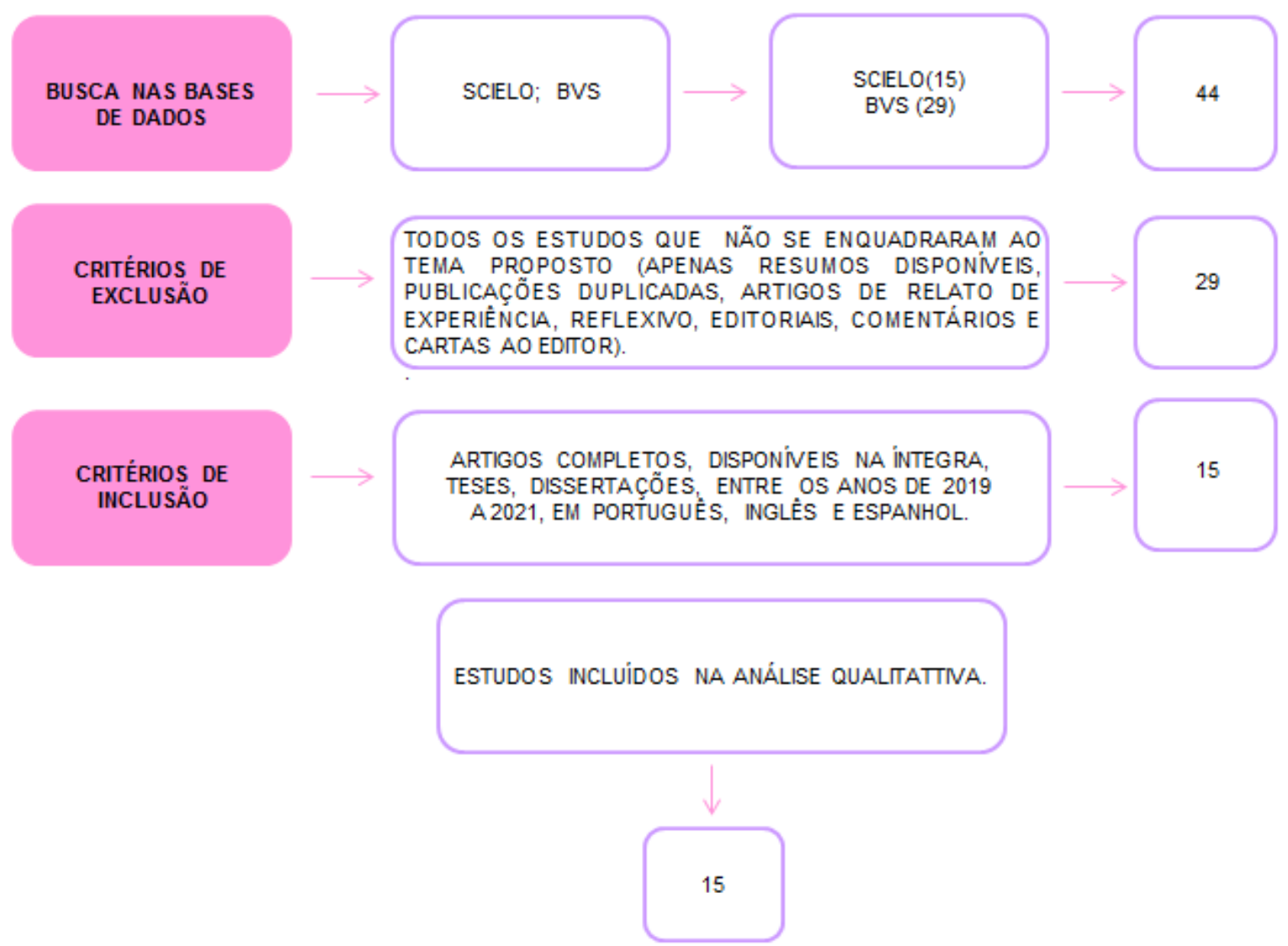

Fonte: Mota et al. (2021).

Os resultados deste trabalho foram obtidos através da análise quantitativa de publicações sobre os principais aspectos envolvidos na infecção por SARS-CoV-2 em pacientes neonatos encontrados nas bases de dados Scientific Electronic Library Online e Biblioteca Virtual em Saúde obtendo 15 artigos na Scielo e 29 artigos na BVS. Foram excluídos 08 artigos da Scielo e 21 da BVS por não se enquadrarem no tema proposto. Foram incluídos na análise qualitativa 07 artigos da Scielo e 08 artigos da BVS entre os anos de 2019 a 2021.

De acordo com a pesquisa realizada nas bases de dados, foi possivel listar os principais títulos e objetivos dos estudos publicados na literatura, utilizados neste trabalho conforme o Quadro 1. 
Quadro 1. Principais títulos e objetivos dos artigos utilizados na pesquisa, Teresina, Brasil, 2021.

\begin{tabular}{|c|c|c|}
\hline $\begin{array}{l}\text { ANO DE } \\
\text { PUBLICAÇÃO }\end{array}$ & TÍTULO & OBJETIVO \\
\hline $\begin{array}{l}\text { Rathberger et al., } \\
2021 .\end{array}$ & $\begin{array}{l}\text { SARS-CoV-2 na gravidez e possível } \\
\text { transferência de imunidade: avaliação dos } \\
\text { níveis de anticorpos maternos e neonatais } \\
\text { peripartais e acompanhamento longitudinal. }\end{array}$ & $\begin{array}{l}\text { Na atual pandemia do Coronavírus } 2 \text { (SARS-CoV-2) } \\
\text { de dificuldade respiratória aguda ainda há grande } \\
\text { incerteza sobre os efeitos de uma infeçãa na gravidez, } \\
\text { especialmente em relação a uma possível transmissão } \\
\text { fetal de anticorpos para SARS-CoV-2 e a longevidade } \\
\text { dessa imunidade. }\end{array}$ \\
\hline Shah; Saugstad, 2021. & $\begin{array}{l}\text { Recém-nascidos em risco de Covid-19 - lições } \\
\text { do ano passado. }\end{array}$ & $\begin{array}{l}\text { Neste artigo, revisamos o desenvolvimento neste } \\
\text { campo ao longo do ano passado e resumimos a } \\
\text { evolução das diretrizes para cuidar de recém-nascidos e } \\
\text { mães com suspeita ou confirmação laboratorial de } \\
\text { infecção por SARS-CoV-2. }\end{array}$ \\
\hline Barbosa et al., 2021. & $\begin{array}{c}\text { Repercussões em recém-nascidos infectados } \\
\text { pelo novo coronavírus durante a gestação: } \\
\text { Revisão integrativa }\end{array}$ & $\begin{array}{c}\text { O objetivo deste estudo é identificar as evidências } \\
\text { disponíveis na literatura sobre as repercussões em } \\
\text { recém-nascidos de mães infectadas pelo coronavírus } \\
\text { durante a gestação. }\end{array}$ \\
\hline $\begin{array}{l}\text { Sheikhahmadi; } \\
\text { Behzadi; Zakaryaei, } \\
2021 .\end{array}$ & $\begin{array}{l}\text { Avaliando a transmissão vertical de COVID-19 } \\
\text { de Mães para recém-nascidos: uma série de } \\
\text { casos iranianos de } 8 \text { Pacientes. }\end{array}$ & $\begin{array}{c}\text { Este estudo apresenta oito mães grávidas com a doença } \\
\text { coronavírus, junto com seus neonatos, que foram } \\
\text { referido ao hospital Besat em Sanandaj, Irã, de Março } \\
\text { a maio de } 2020 \text {. }\end{array}$ \\
\hline $\begin{array}{l}\text { Oliveira, M. A. D. et } \\
\text { al., } 2021 .\end{array}$ & $\begin{array}{l}\text { Recomendações para assistência perinatal no } \\
\text { contexto da pandemia de COVID19. }\end{array}$ & $\begin{array}{l}\text { O presente estudo objetivou apresentar as } \\
\text { recomendações científicas para a atenção perinatal no } \\
\text { contexto da pandemia de COVID-19. }\end{array}$ \\
\hline Goés et al., 2020. & $\begin{array}{l}\text { Boas Práticas No Cuidado Ao Recém-nascido } \\
\text { Em Tempos De Covid-19: Revisão Integrativa. }\end{array}$ & $\begin{array}{l}\text { Identificar evidências científicas sobre boas práticas no } \\
\text { cuidado ao recém-nascido, da sala de parto ao } \\
\text { domicílio, em tempos de COVID-19. }\end{array}$ \\
\hline Freitas et al., 2020. & $\begin{array}{l}\text { Medidas de prevenção e controle de infecção } \\
\text { neonatal por COVID-19: revisão de escopo. }\end{array}$ & $\begin{array}{l}\text { Identificar junto à literatura as medidas de prevenção e } \\
\text { controle de infecção neonatal por COVID-19. }\end{array}$ \\
\hline Sankaran et al., 2021. & $\begin{array}{l}\text { Infecção Perinatal SARS-CoV-2 e Neonatal } \\
\text { COVID-19: Atualização A } 2021 .\end{array}$ & $\begin{array}{c}\text { Descrever abordagens de manejo perinatal para } \\
\text { mulheres grávidas para melhorar os resultados em } \\
\text { mães e recém-nascidos. }\end{array}$ \\
\hline Duran et al., 2020. & $\begin{array}{l}\text { COVID-19 e saúde do recém-nascido: revisão } \\
\text { sistemática. }\end{array}$ & $\begin{array}{l}\text { Descrever os resultados perinatais e neonatais em } \\
\text { recém-nascidos expostos ao SARS-CoV- } 2 \text {. }\end{array}$ \\
\hline Malik et al., 2021. & $\begin{array}{l}\text { Características clínicas, manejo e resultados de } \\
\text { curto prazo de recém-nascidos de mães com } \\
\text { COVID-19 em um hospital terciário na Índia. }\end{array}$ & $\begin{array}{l}\text { Descrever as características clínicas, o manejo e os } \\
\text { resultados de curto prazo de neonatos com SARS- } \\
\text { CoV-2 nascidos de mães com COVID-19 em um } \\
\text { hospital terciário em Mumbai, Índia. }\end{array}$ \\
\hline Yee et al., 2020. & $\begin{array}{l}\text { Manifestações clínicas e resultados perinatais } \\
\text { de mulheres grávidas com COVID-19: uma } \\
\text { revisão sistemática e meta-análise. }\end{array}$ & $\begin{array}{l}\text { Avaliar o impacto do COVID-19 em mulheres } \\
\text { grávidas. }\end{array}$ \\
\hline Chen et al., 2020. & $\begin{array}{l}\text { Características clínicas e potencial de } \\
\text { transmissão vertical intrauterina da infecção por } \\
\text { COVID-19 em nove gestantes: uma revisão } \\
\text { retrospectiva de prontuários médicos. }\end{array}$ & $\begin{array}{c}\text { Este estudo teve como objetivo avaliar as } \\
\text { características clínicas do COVID-19 na gravidez e o } \\
\text { potencial de transmissão vertical intrauterina da } \\
\text { infecção pelo COVID-19. }\end{array}$ \\
\hline Melo; Araújo, 2020. & $\begin{array}{l}\text { Infecção por COVID-19 em mulheres grávidas, } \\
\text { parto prematuro, peso ao nascer e transmissão } \\
\text { vertical: uma revisão sistemática e meta- }\end{array}$ & $\begin{array}{l}\text { Analisar se a infecção por COVID- } 19 \text { em mulheres } \\
\text { grávidas está relacionada ao nascimento prematuro e } \\
\text { peso ao nascer, e para resumir os resultados }\end{array}$ \\
\hline
\end{tabular}




\begin{tabular}{|c|c|c|}
\hline & análise. & $\begin{array}{c}\text { diagnósticos de neonatos nascidos de mães com } \\
\text { infecção por COVID-19 para investigar a possibilidade } \\
\text { de transmissão vertical. }\end{array}$ \\
\hline Baquero et al., 2020. & Sepse neonatal tardia devido a SARS CoV-2. & $\begin{array}{c}\text { Relatar três casos de recém-nascidos que foram } \\
\text { atendidos durante a fase de mitigação no serviço de } \\
\text { emergência de um hospital materno em Barranquilla, } \\
\text { Colômbia, por temperatura elevada e estado geral } \\
\text { comprometido. }\end{array}$ \\
\hline Almeida et al., 2021. & $\begin{array}{c}\text { Manifestações clínicas do Covid-19 na } \\
\text { população pediátrica e neonatal. }\end{array}$ & $\begin{array}{c}\text { O objetivo desse trabalho é reunir informações para o } \\
\text { melhor entendimento da doença nessa faixa etária. }\end{array}$ \\
\hline
\end{tabular}

Fonte: SCIELO e BVS.

Desta forma, foi possível listar os principais resultados e conclusões dos estudos publicados na literatura, conforme o

Quadro 2.

Quadro 2. Resultados e conclusões dos artigos utilizados, Teresina, Brasil, 2021.

\begin{tabular}{|c|c|c|}
\hline PUBLICAÇ̃̃OO & RESULTADOS & CONCLUSÕES \\
\hline $\begin{array}{l}\text { Rathberger et al., } \\
2021 .\end{array}$ & $\begin{array}{l}\text { Mulheres grávidas e recém-nascidos são um } \\
\text { grupo particularmente suscetível a complicações } \\
\text { de infecções respiratórias. Portanto, durante a } \\
\text { atual pandemia, é importante explorar os } \\
\text { mecanismos de imunidade neste grupo } \\
\text { vulnerável. Neste estudo de } 15 \text { mulheres que } \\
\text { foram testadas para SARS-CoV-2 positivas } \\
\text { durante a gravidez, todas, exceto uma paciente, } \\
\text { apresentaram cursos leves a assintomáticos de } \\
\text { COVID-19 e não relataram sintomas residuais } \\
\text { no acompanhamento pós-parto. }\end{array}$ & $\begin{array}{l}\text { A possível transferência transplacentária de proteção } \\
\text { de anticorpos e sua longevidade são particularmente } \\
\text { relevantes no que diz respeito a uma possível } \\
\text { vacinação de mulheres grávidas contra a SARS- } \\
\text { CoV-2. A vacinação contra SARS-CoV-2 (assim } \\
\text { que for considerada segura na gravidez) pode ser } \\
\text { aconselhável para ser administrada no primeiro ou } \\
\text { segundo trimestre para permitir a possível } \\
\text { transmissão de imunidade à criança. }\end{array}$ \\
\hline Shah; Saugstad, 2021. & $\begin{array}{l}\text { Embora a morbidade materna possa ser grave, a } \\
\text { transmissão vertical é rara. Huntely et } \\
\text { al. revisou } 99 \text { artigos e não encontrou } \\
\text { transmissão vertical confirmada. A transmissão } \\
\text { horizontal de SARS-CoV-2 para o recém- } \\
\text { nascido parece ocorrer principalmente por meio } \\
\text { de gotículas respiratórias quando os neonatos } \\
\text { são expostos a mães ou outros cuidadores com } \\
\text { infecção por SARS-CoV-2. }\end{array}$ & $\begin{array}{c}\text { Embora muito tenha sido aprendido sobre as práticas } \\
\text { ideais de maternidade durante a pandemia de } \\
\text { COVID-19, menos conhecidos são os aspectos } \\
\text { psicossociais e os efeitos de longo prazo em } \\
\text { neonatos de mães com infecção por SARS-CoV-2 e } \\
\text { esforços contínuos para coletar dados devem } \\
\text { continuar. }\end{array}$ \\
\hline Barbosa et al., 2021. & $\begin{array}{l}\text { Ao pensar as formas de transmissão do vírus } \\
\text { durante o período gestacional, deve-se levar em } \\
\text { consideração a correlação da infecção por } \\
\text { COVID-19 durante a gestação, com o parto } \\
\text { prematuro e o peso ao nascer, além da } \\
\text { possibilidade de transmissão vertical. Com isso, } \\
\text { verificou-se casos com níveis de reagentes IgM } \\
\text { encontrados precocemente em neonatos, o que } \\
\text { somente é possível devido a produção de IgM } \\
\text { no feto em resposta a transmissão vertical do } \\
\text { SARS-CoV-2. }\end{array}$ & $\begin{array}{c}\text { Ao analisar as evidências sobre as repercussões em } \\
\text { RN infectados pela COVID-19, observa-se a } \\
\text { escassez de estudos retrospectivos e que possam } \\
\text { levantar as consequências em longo prazo da ação } \\
\text { viral no organismo de neonatos e crianças } \\
\text { infectadas. }\end{array}$ \\
\hline Sheikhahmadi; & $\begin{array}{l}\text { No presente estudo, embora os neonatos } \\
\text { infectados com SARS-CoV-2 teve dificuldade } \\
\text { respiratória, nenhum deles foi observada febre, } \\
\text { má alimentação, vômito ou diarréia. De acordo }\end{array}$ & $\begin{array}{l}\text { Em conclusão, os resultados mostraram que } \\
\text { COVID-19 tem bom prognóstico em neonatos sem } \\
\text { problemas congênitos. Recomenda-se o uso de } \\
\text { suporte adequado e antibioticoterapia para neonatos }\end{array}$ \\
\hline
\end{tabular}




\begin{tabular}{|c|c|c|}
\hline $\begin{array}{l}\text { Behzadi; Zakaryaei, } \\
2021 .\end{array}$ & $\begin{array}{l}\text { com os resultados de Zeng et al, recém-nascidos } \\
\text { são mais sintomáticos de prematuridade, asfixia } \\
\text { e sepsia, do que a infecção SARS-CoV-2. }\end{array}$ & infectados com COVID-19. \\
\hline $\begin{array}{l}\text { Oliveira, M. A. D. et } \\
\text { al., } 2021 .\end{array}$ & $\begin{array}{l}\text { As pesquisas descrevem algumas das alterações } \\
\text { necessárias durante a rotina de cuidado prestado } \\
\text { ao binômio mãe-bebê, desde as consultas pré- } \\
\text { natais, a admissão no serviço hospitalar, ao } \\
\text { puerpério e aos cuidados com o recém-nascido. }\end{array}$ & $\begin{array}{l}\text { É necessária a continuidade pré-natal, incluindo a } \\
\text { modalidade de teleconsultas. Recomenda-se } \\
\text { internação em casos específicos para rigoroso } \\
\text { monitoramento, devendo-se interromper a gestação } \\
\text { em quadro de gravidade materna ou fetal, por via } \\
\text { cesariana. A amamentação deve ser estimulada, } \\
\text { desde que cuidados maternos sejam tomados. }\end{array}$ \\
\hline Goés et al., 2020. & $\begin{array}{l}\text { Dezenove estudos compuseram a amostra final, } \\
\text { dos quais emergiram cinco categorias analíticas } \\
\text { sobre as boas práticas no cuidado ao recém- } \\
\text { nascido: gestantes e puérperas com suspeita da } \\
\text { COVID-19; gestantes e puérperas com } \\
\text { confirmação da COVID-19; recém-nascido com } \\
\text { suspeita da COVID-19; recém-nascido com } \\
\text { confirmação da COVID-19; e prevenção da } \\
\text { transmissão horizontal da COVID-19 ao recém- } \\
\text { nascido. }\end{array}$ & $\begin{array}{c}\text { Assim sendo, são necessárias novas pesquisas, } \\
\text { especialmente com nível de evidência forte, para a } \\
\text { formulação de diretrizes assistenciais a esse grupo } \\
\text { populacional que contribuam para a redução da } \\
\text { morbimortalidade neonatal e o desenvolvimento } \\
\text { infantil saudável e harmonioso durante e pós- } \\
\text { pandemia. }\end{array}$ \\
\hline Freitas et al., 2020. & $\begin{array}{l}\text { A partir dos resultados, foi possível verificar } \\
\text { que as publicações sobre as medidas de } \\
\text { prevenção e controle de infecção neonatal por } \\
\text { COVID-19 ainda são incipientes, com poucos } \\
\text { estudos originais desenvolvidos até então, e a } \\
\text { maioria originária da China, primeiro país a } \\
\text { identificar a doença. Obviamente, isso ocorre } \\
\text { devido a doença ser recente. }\end{array}$ & $\begin{array}{l}\text { Este estudo contribui para o corpo de conhecimento } \\
\text { dos enfermeiros e demais profissionais da saúde no } \\
\text { tocante às principais medidas de prevenção e } \\
\text { controle de infecção neonatal por COVID-19. }\end{array}$ \\
\hline Sankaran et al., 2021. & $\begin{array}{c}\text { Os médicos neonatais devem assistir aos partos } \\
\text { com base nas políticas específicas do hospital / } \\
\text { centro. O COVID-19 materno por si só não é } \\
\text { uma indicação específica para assistir a um } \\
\text { parto. }\end{array}$ & $\begin{array}{l}\text { Os cuidados maternos e neonatais durante a } \\
\text { pandemia COVID-19 têm sido um desafio para os } \\
\text { médicos. Isso se deve à vulnerabilidade dessas } \\
\text { populações, à falta de evidências de alta qualidade } \\
\text { em estratégias de tratamento e resultados de } \\
\text { pacientes infectados. }\end{array}$ \\
\hline Duran et al., 2020. & $\begin{array}{c}\text { De } 256 \text { publicações identificadas, } 20 \\
\text { preencheram os critérios de inclusão e incluíram } \\
\text { dados de resultados neonatais para } 222 \text { recém- } \\
\text { nascidos cujas mães eram suspeitas ou } \\
\text { confirmadas como soropositivas para SARS- } \\
\text { CoV-2 no período perinatal (17 estudos) ou de } \\
\text { recém-nascidos encaminhados ao hospital com } \\
\text { infecção / pneumonia ( } 3 \text { estudos). }\end{array}$ & $\begin{array}{l}\text { Dada a escassez de estudos neste momento, a } \\
\text { transmissão vertical não pode ser confirmada ou } \\
\text { negada. A literatura atual não apóia a abstenção da } \\
\text { amamentação nem a separação de mães e recém- } \\
\text { nascidos. Mais evidências e redes de coleta de } \\
\text { dados, particularmente nas Américas, são } \\
\text { necessárias para estabelecer diretrizes e } \\
\text { recomendações definitivas. }\end{array}$ \\
\hline Malik et al., 2021. & $\begin{array}{l}\text { A infecção por SARS-CoV-2 foi detectada em } \\
6,3 \% \text { dos recém-nascidos de mães com COVID- } \\
\text { 19. Não foram observadas diferenças } \\
\text { significativas entre a maturidade na gestação, o } \\
\text { peso ao nascer e o sexo dos recém-nascidos } \\
\text { infectados e não infectados com SARS-CoV-2. } \\
\text { O risco de sepse foi } 4,09 \text { [intervalo de confiança } \\
\text { de } 95 \% \text { (IC 95\%) } 1,28-13,00 \text { ] vezes maior nos } \\
\text { neonatos com SARS-CoV-2 em comparação } \\
\text { com o grupo não infectado ( } p=0,031) \text {. }\end{array}$ & $\begin{array}{l}\text { A infecção neonatal por SARS-CoV-2 é observada } \\
\text { em quase } 6 \% \text { dos neonatos nascidos de mães com } \\
\text { COVID-19 perinatal. Há um risco maior de } \\
\text { resultados adversos, como sepse neonatal e morte } \\
\text { nos infectados com SARS-CoV-2 em comparação } \\
\text { com os neonatos não infectados. }\end{array}$ \\
\hline Yee et al., 2020. & $\begin{array}{l}\text { A gravidez e os resultados perinatais de } \\
\text { pacientes grávidas infectadas pelo SARS-CoV- } \\
\text { 2. Cerca de 30\% das mulheres grávidas com } \\
\text { COVID-19 tiveram parto prematuro, enquanto a } \\
\text { ruptura prematura das membranas e sofrimento }\end{array}$ & $\begin{array}{l}\text { A presente meta-análise apresenta algumas } \\
\text { limitações que devem ser consideradas na } \\
\text { interpretação dos resultados. As manifestações } \\
\text { clínicas detalhadas não estavam disponíveis nos }\end{array}$ \\
\hline
\end{tabular}




\begin{tabular}{|c|c|c|}
\hline & $\begin{array}{l}\text { fetal foram observados em cerca de } 2 \% \text {. O peso } \\
\text { médio ao nascer foi de } 2.855,9 \mathrm{~g} \text { (IC95\% } \\
2634,9-3076,9 \mathrm{~g} \text { ) e a prevalência de } \\
\text { nascimentos pequenos para a idade gestacional } \\
\text { foi estimada em } 17,4 \% \text { (IC95\% 0-56,0\%) }\end{array}$ & $\begin{array}{l}\text { estudos selecionados. Além disso, as associações } \\
\text { entre características clínicas e resultados não foram } \\
\text { fornecidas. }\end{array}$ \\
\hline Chen et al., 2020. & $\begin{array}{l}\text { Todos os nove pacientes tiveram uma cesariana } \\
\text { no terceiro trimestre. Sete pacientes } \\
\text { apresentaram febre. Outros sintomas, incluindo } \\
\text { tosse (em quatro de nove pacientes), mialgia } \\
\text { (em três), dor de garganta (em dois) e mal-estar } \\
\text { (em dois), também foram observados. O } \\
\text { sofrimento fetal foi monitorado em dois casos. }\end{array}$ & $\begin{array}{l}\text { As características clínicas da pneumonia por } \\
\text { COVID-19 em mulheres grávidas foram } \\
\text { semelhantes às relatadas para pacientes adultas não } \\
\text { grávidas que desenvolveram pneumonia por } \\
\text { COVID-19. }\end{array}$ \\
\hline Melo; Araújo, 2020. & $\begin{array}{l}\text { Resultados positivos para SARS-CoV-2 foram } \\
\text { encontrados em } 16 \text { neonatos. Verificações } \\
\text { repetidas na mesma amostra de esfregaço da } \\
\text { garganta mostraram que o resultado era falso } \\
\text { positivo para um desses neonatos nascidos na } \\
\text { China e o vírus era indetectável no líquido } \\
\text { amniótico e no sangue do cordão umbilical para } \\
\text { este recém-nascido. }\end{array}$ & $\begin{array}{l}\text { O COVID-19 em mulheres grávidas pode não estar } \\
\text { associado à ocorrência de partos prematuros ou ao } \\
\text { peso ao nascer de recém-nascidos, no entanto, as } \\
\text { evidências até o momento são muito incertas. } \\
\text { Alguns relatórios sugerem que a transmissão vertical } \\
\text { de SARS-CoV-2 para recém-nascidos é possível, } \\
\text { mas a evidência ainda é incerta. }\end{array}$ \\
\hline Baquero et al., 2020. & $\begin{array}{c}\text { Durante o curso clínico, desenvolveram } \\
\text { sintomas gastrointestinais sem apresentar } \\
\text { manifestações respiratórias. Não estavam } \\
\text { epidemiologicamente vinculados a um contato } \\
\text { com suspeita de ser um caso de COVID-19 e } \\
\text { suas mães não apresentavam sintomas } \\
\text { respiratórios desde que a emergência de saúde } \\
\text { pública em nosso país foi declarada } 45 \text { dias } \\
\text { antes. }\end{array}$ & $\begin{array}{l}\text { Os mecanismos fisiopatológicos da COVID-19 } \\
\text { incluem infecção e envolvimento do trato } \\
\text { gastrointestinal. Recém-nascidos com manifestações } \\
\text { gastrointestinais, febris ou afebris, avaliados quanto } \\
\text { a sintomas sugestivos de sepse tardia durante a } \\
\text { pandemia, devem ser rastreados para infecção por } \\
\text { SARS CoV-2, independentemente de haver ou não } \\
\text { uma ligação epidemiológica comprovada. }\end{array}$ \\
\hline Almeida et al., 2021. & $\begin{array}{c}\text { Diferentemente dos adultos, as crianças } \\
\text { infectadas pelo SARS-CoV-2 apresentam } \\
\text { formas clínicas leves ou assintomáticas na } \\
\text { maior parte dos casos descritos na literatura. } \\
\text { Com relação à infecção, as crianças possuem a } \\
\text { mesma probabilidade do que os adultos, } \\
\text { contudo, a apresentação dos sintomas é que os } \\
\text { diferencia. }\end{array}$ & $\begin{array}{c}\text { Os casos de SARS-Cov-2 na população pediátrica } \\
\text { correspondem a menos de } 5 \% \text { de todos os casos } \\
\text { envolvendo esse vírus reportados pela comunidade } \\
\text { científica. }\end{array}$ \\
\hline
\end{tabular}

Fonte: SCIELO e BVS.

De acordo com os artigos científicos encontrados nota-se que a grande maioria dos estudos possui fraco nível de evidência, o que é esperado devido à contemporaneidade da pandemia causada pelo SARS-CoV-2. Mas podemos observar que a COVID-19 é uma doença causada pela infecção com o vírus SARS-CoV-2 detectada pela primeira vez em Wuhan, China, em dezembro de 2019. A transmissão vertical desta doença ainda não foi totalmente esclarecida. O sistema imunológico imaturo dos neonatos permite a transmissão vertical do vírus. Recém-nascidos que são muito suscetíveis à infecção por COVID-19 devem ser mantidos em incubadora (Sheikhahmadi; Behzadi; Zakaryaei, 2021).

Observa-se que ao contrário dos adultos e idosos, as crianças infectadas pelo vírus desenvolvem formas mais graves da doença raramente. Eles geralmente são assintomáticos ou apresentam sintomas leves, incluindo febre, tosse e eritema na faringe. Entre os sintomas considerados menos prevalentes estão à diarreia, coriza, fadiga, falta de ar e congestão nasal. Em recém-nascidos, os sintomas podem ser mais distintos. Em alguns casos menos prevalentes, podem ocorrer consequências graves, como icterícia, alterações da função hepática e sepse neonatal tardio. Portanto, os sintomas inespecíficos podem ser confundidos com outras doenças comuns da infância. O reconhecimento precoce de sinais de dificuldade respiratória pode garantir que os profissionais tomem decisões clínicas, evitando que a doença se agrave (Barbosa et al., 2021). 
Gestantes e puérperas são considerados grupos de maior risco clínico para a COVID-19, visto que apresentam alterações no mecanismo imunológico e são mais sensíveis a hipóxia. Essa situação é associada a um aumento de desfechos negativos, como internações e óbitos, e representa risco maior de transmissão da infecção para o RN. A transmissão pós-parto ou horizontal do vírus para o RN é mais frequente e agrava-se quando a mãe é sintomática para COVID-19 devido a maior probabilidade do contato com gotículas ou material biológico infectado. Nessa situação é necessário que haja protocolos na assistência para redução do risco de infecção na sala de parto (Oliveira, et al., 2021; Chen et al., 2020).

A transmissão do vírus para o RN pode acontecer por conta do contato de forma direta através da exposição a gotículas respiratórias de pessoas infectadas ou pelo contato indireto no ambiente ou com objetos utilizados pelo indivíduo infectado. Ainda há muito a se entender sobre a transmissão do vírus da COVID-19, o tratamento e as demais condições relacionadas aos pacientes neonatais, por consequência as investigações estão sendo realizadas mundialmente. A prevenção e o controle dessa infecção em pacientes neonatos é imprescindível nos serviços de saúde, especialmente por essa ser uma população com o sistema imune imaturo, sendo vista como de risco (Freitas et al., 2020).

Há uma preocupação levantada sobre o risco que a infecção por COVID-19 pode representar para o feto. Se a propagação vertical do vírus é possível e quais consequências ela teria, ainda não está totalmente esclarecido, mas o risco parece relativamente pequeno. No entanto, mesmo com estudos inovadores nos últimos meses, as consequências do longo prazo da infecção por SARS-CoV-2 durante a gravidez em mães e crianças, incluindo a possível transferência imunológica, ainda são amplamente desconhecidas (Rathberger et al., 2021; Melo; Araújo, 2020).

Com relação aos pacientes RN, atualmente não existem informações suficientes sobre as manifestações clínicas da COVID-19. Estudos mostram que as apresentações da infecção são consideradas menos graves nas crianças do que em pessoas adultas, e que as crianças menores de um ano podem apresentar alterações mais graves do que as crianças com mais idade. Os recém-nascidos merecem uma atenção especial, devido à imaturidade do seu sistema imunológico, tornando-os mais favoráveis à infecção pelo vírus, além da probabilidade de transmissão vertical da mãe para o filho (Freitas et al., 2020).

Os RN de mães positivas para o SARS-CoV-2 que tiveram contato próximo com algum indivíduo com suspeita ou diagnostico confirmado para a doença e/ou moram ou viajaram para locais com elevados números de casos são considerados de elevado risco para a COVID-19. As manifestações clínicas dos RN infectados, geralmente, não são específicas. Quando ocorrem, se destacam, principalmente, em bebês com prematuridade, com problemas cardiovasculares e gastrointestinais, instabilidade térmica e distúrbios respiratórios. Em casos mais graves, eles podem desenvolver de forma rápida a Síndrome do Desconforto Respiratório Agudo. Toda via, na medida em que a função do sistema imunológico neonatal é imatura, deve-se ter atenção ao risco de COVID-19 neonatal e à possibilidade da condição se tornar grave (Goés et al., 2020; Almeida et al., 2021).

Estudos descrevem ser possível a transmissão vertical visto que após realização de exames para identificação do vírus SARS-CoV-2 em amostra biológica de neonatos obteve-se resultado positivo. Um relato de caso que foi realizado na França, descreveu a presença de SARS-CoV-2 em todas as amostras laboratoriais, obtidos a partir do líquido amniótico (LA), coletado antes da ruptura das membranas, sangue da placenta e sangue do RN, coletado nas primeiras 12 horas de vida pós parto cesáreo de mãe positiva para COVID-19. Foi possível observar sinais de inflamação na placenta característicos da infecção e células inflamatórias no líquido cefalorraquidiano do neonato (Oliveira, M. A. D. et al., 2021).

Atualmente, não há evidências convincentes de que o SARS-CoV-2 possa ser transmitido de uma mãe infectada para os recém-nascidos através do leite materno; pelo contrário, o leite materno pode ajudar a fornecer anticorpos protetores contra a infecção pelo SARS-CoV-2. Se a saúde da mãe permitir, os benefícios nutricionais, imunológicos e de desenvolvimento da amamentação superam o risco potencial de transmissão, porque os bebês geralmente sofrem de doenças leves. Os recémnascidos têm maior probabilidade de adquirir infecções por transmissão horizontal de mães infectadas ou outros profissionais de saúde; portanto, a importância de manter uma higiene respiratória adequada quando uma pessoa infectada entra em contato 
com um recém-nascido não pode ser subestimada (Sankaran et al., 2021; Yee et al., 2020).

Embora alguns estudos tenham refutado essa possibilidade, existe uma probabilidade razoável de transmissão vertical do SARS-CoV-2 após o nascimento do RN. Além disso, a infecção por SARS-CoV-2 perinatal afeta a saúde materna e da placenta, o que pode afetar o curso clínico dos recém-nascidos pós-natal (Malik et al., 2021).

Diferente de adultos, os RN apresentam um sistema imunológico imaturo, e este fato associado à transferência passiva de anticorpos IgG maternos e menor expressão de ACE-2 podem fazer com que bebês e crianças tenham menos inflamações, doenças mais brandas e recuperação mais rápida. Em uma revisão sistemática, foi relatado que os recém-nascidos são mais gravemente doentes (12\% dos bebês infectados) em comparação com as crianças mais velhas (3\% das crianças mais velhas requerem cuidados intensivos). Bebês com SARS-CoV-2 positivo devem ser monitorados clinicamente e isolados (Sankaran et al., 2021).

A sepse neonatal tardia é um considerado uma das principais manifestações que ocorre na infecção neonatal com COVID-19. A transmissão vertical da infecção ainda não foi documentada, mas os estudos mostram a probabilidade disso acontecer. É de extrema importância que os cuidados tanto com a transmissão de forma horizontal (mãe para filho), quanto com a transmissão vertical sejam realizados, uma vez que podem levar ao parto prematuro, a ruptura das membranas de forma prematura e ao sofrimento fetal (Barbosa et al., 2021; Baquero et al., 2020).

O SARS-CoV-2 é um novo vírus que requer uma resposta rápida dos serviços de saúde, devido a sua alta transmissibilidade e evidências críticas estão sendo coletadas e verificadas em estudos científicos. Embora o foco principal seja em grupos vulneráveis, especialmente idosos e pessoas com doenças de base, as mulheres grávidas e os recém-nascidos também podem enfrentar maiores riscos. Até o momento, existem uma série de casos e relatos de casos limitados sobre COVID-19 durante a gravidez, sobre possível transmissão de mãe para filho e infecções neonatais e infantis. A COVID-19 em recém-nascidos foi descrita como uma doença leve, entretanto, existe preocupações quanto às complicações da infecção nos recém-nascidos, tanto em termos de impacto como de cuidados adequados. Ao formular recomendações para esses grupos, é importante entender as questões relacionadas à essas questões perinatais (Duran et al., 2020).

Estudos mostram que é alta a possibilidade de transmissão materno-fetal do SARS-CoV-2 devido a várias razões, como a expressão do receptor ACE-2 na placenta. Entretanto, a menor resposta dentro da célula induzida por ACE-2 nas células epiteliais alveolares e a presença de cadeias alfa e gama na hemoglobina fetal podem manifestar uma resposta protetora em neonatos. Apesar dessa hipótese, infecção grave e resultados adversos são relatados em recém-nascidos e a ocorrência de transmissão vertical é demonstrada em alguns dos estudos científicos (Malik et al., 2021).

\section{Conclusão}

A infecção SARS-Cov-2 em pacientes neonatais representam menos de 5\% de todos os casos que envolvem esses vírus mencionados em estudos científicos. A maioria das crianças e neonatos que apresentam a COVID-19 são considerados como assintomáticos ou possuem manifestações clínicas mais leves, tornando-se transmissores da infecção para o restante da população.

Ao analisar os estudos científicos sobre a infecção por SARS-CoV-2 em pacientes neonatos, observa-se a escassez de trabalhos que possam mostrar as ações do vírus em longo prazo no organismo desses pacientes. Não há resultados claros sobre a infecção de RN pela COVID-19, o que dificulta a assistência desses pacientes. Nos estudos que foram relatados, ocorrem diferentes resultados clínicos com pacientes recém-nascidos que variam desde assintomáticos até casos graves e fatais com importante comprometimento respiratório e sistêmico. Deve-se levar em consideração o surgimento recente do vírus e as diversas formas de tratamento e protocolos na assistência prestada pelos serviços de saúde. 
Apesar de não ser possível presumir a repercussão para os pacientes neonatais, é possível evitar a mortalidade com adoção de protocolos já estabelecidos. É primordial a busca constante em esclarecer os mecanismos, e as formas de transmissão nesses pacientes, em vista disso, a realização de novos estudos científicos mais completos que abordem esta temática faz-se necessário. Espera-se que sejam publicadas novas evidências científicas, de forma que a assistência perinatal no contexto da pandemia pela a infecção por SARS-CoV-2 aconteça de forma segura e livre de complicações e desfechos desfavoráveis ao recém-nascido, bem como a mãe.

\section{Referências}

Almeida, S. L. A. C., Chávare, J., Halfeld, J. C., dos Santos Costa, J. F., Parizzi, J. F., Primavera, J. M., \& de Oliveira, L. F. (2021). Manifestações clínicas do Covid-19 na população pediátrica e neonatal. Brazilian Journal of Health Review, 4(2), 4582-4591.

Baquero, H., Venegas, M. E., Velandia, L., Neira, F., \& Navarro, E. (2020). Neonatal late-onset infection with SARS CoV-2. Biomédica, 40, 44-49.

Barbosa, A. C. S., da Costa, C. M., da Silva Santos, D. T., Fonseca, I. C. V., Freitas, L. C. D., Fonseca, L. B., \& Oliveira, M. M. (2021). Repercussões em recém-nascidos infectados pelo novo coronavírus durante a gestação: Revisão integrativa. Research, Society and Development, 10(8), e31510817364e31510817364.

Brasil. (2020). Atenção à saúde do recém-nascido no contexto da infecção pelo novo coronavírus. Nota Técnica $\mathrm{N}^{\circ}$ 6/2020 COCAM/CGCIVI/DAPES/SAPS/MS.

Cavalcante, J. R., Cardoso-dos-Santos, A. C., Bremm, J. M., Lobo, A. D. P., Macário, E. M., Oliveira, W. K. D., \& França, G. V. A. D. (2020). COVID-19 no Brasil: evolução da epidemia até a semana epidemiológica 20 de 2020. Epidemiologia e Serviços de Saúde, 29.

Chen, H., Guo, J., Wang, C., Luo, F., Yu, X., Zhang, W., \& Zhang, Y. (2020). Clinical characteristics and intrauterine vertical transmission potential of COVID-19 infection in nine pregnant women: a retrospective review of medical records. The lancet, 395(10226), 809-815.

Duran, P., Berman, S., Niermeyer, S., Jaenisch, T., Forster, T., Gomez Ponce de Leon, R., \& Serruya, S. (2020). COVID-19 and newborn health: systematic review. Revista Panamericana de Salud Pública, 44-54.

Freitas, B. H. B. M. D., Alves, M. D. D. S. M., \& Gaíva, M. A. M. (2020). Medidas de prevenção e controle de infecção neonatal por COVID-19: revisão de escopo. Revista Brasileira de Enfermagem, 73.

Góes, F. G. B., Santos, A. S. T. D., Lucchese, I., Silva, L. J. D., Silva, L. F. D., \& Silva, M. D. A. (2020). Boas práticas no cuidado ao recém-nascido em tempos de covid-19: revisão integrativa. Texto \& Contexto-Enfermagem, 29.

Malik, S., Surve, S., Wade, P., Kondekar, S., Sawant, V., Shaikh, M., \& Gajbhiye, R. K. (2021). Clinical characteristics, management, and short term outcome of neonates born to mothers with COVID-19 in a tertiary care hospital in India. Journal of Tropical Pediatrics, 67.

Melo, G. C. D., \& Araújo, K. C. G. M. D. (2020). COVID-19 infection in pregnant women, preterm delivery, birth weight, and vertical transmission: a systematic review and meta-analysis. Cadernos de saude publica, 36, e00087320.

Oliveira, C. E. D. S., Moura, M. Á. P., Dantas, A. L. B., Gouveia, M. T. D. O., \& Mascarenhas, V. H. A. (2021). Assistência ao recém-nascido na sala de parto durante a pandemia de COVID-19. Acta Paulista de Enfermagem, 34.

Oliveira, M. A. D., Silva, N. É. F., Pereira, J. D. C. N., Oliveira, M. A. D., Silva, S. L. D., Caminha, M. D. F. C., \& Cruz, R. D. S. B. L. C. (2021). Recomendações para assistência perinatal no contexto da pandemia de COVID-19. Revista Brasileira de Saúde Materno Infantil, $21,65-75$.

Rathberger, K., Häusler, S., Wellmann, S., Weigl, M., Langhammer, F., Bazzano, M. V., \& Malfertheiner, S. F. (2021). SARS-CoV-2 in pregnancy and possible transfer of immunity: assessment of peripartal maternal and neonatal antibody levels and a longitudinal follow-up. Journal of Perinatal Medicine, 49(6) 702-708.

Sankaran, D., Nakra, N., Cheema, R., Blumberg, D., \& Lakshminrusimha, S. (2021). Infecção sars-cov-2 perinatal e covid-19 neonatal: uma atualização de 2021. NeoReviews, 22 (5), e284-e295.

Shah, MD, e Saugstad, OD (2021). Recém-nascidos em risco de Covid-19 - lições do ano passado. Journal of Perinatal Medicine, 49 (6) 643-649.

Sheikhahmadi, S., Behzadi, S., \& Zakaryaei, F. (2021). Evaluating Vertical Transmission of COVID-19 from Mothers to Neonates: An Iranian Case Series of 8 Patients. Archives of Iranian Medicine, 24(5), 405-408.

Yee, J., Kim, W., Han, J. M., Yoon, H. Y., Lee, N., Lee, K. E., \& Gwak, H. S. (2020). Clinical manifestations and perinatal outcomes of pregnant women with COVID-19: a systematic review and meta-analysis. Scientific reports, 10(1), 1-7. 